\title{
A Janus-Headed Polyp: Adenoma and Carcinoma with a Single Stalk
}

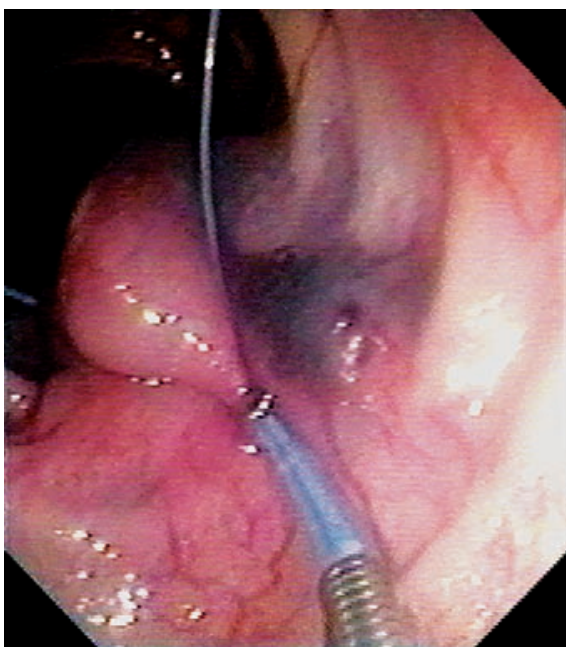

Figure 1 A 70-year-old patient was admitted to hospital due to lower gastrointestinal bleeding from a biopsy site in a large polyp in the sigmoid colon. A polypectomy was performed after placement of an Endoloop. Histopathological examination revealed a tubular villous adenoma and an adenocarcinoma, with the two tumors growing out of a single pedicle.

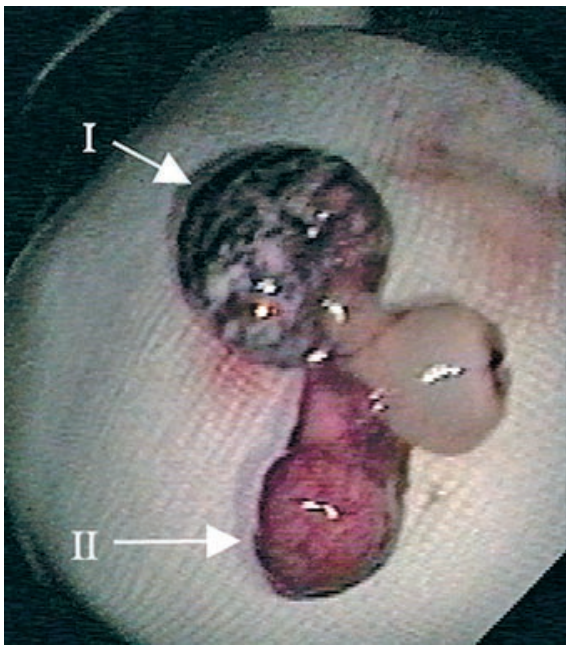

Figure 4 The resection specimen shows a two-headed polyp growing out of a single stalk. The first head (the adenocarcinoma, I) measured $1.5 \times 1.5 \mathrm{~cm}$, the second head (the adenoma, II) was $1.0 \times 1.0 \mathrm{~cm}$, and the pedicle was $1.3 \times 0.7 \mathrm{~cm}$. So far as we are aware, this is the first description of an adenocarcinoma and an adenoma growing out of a single stalk. We have termed it "Janus-headed" after the Roman god Janus, the double-faced god of entrances and exits, whose image was often placed in doorways and who symbolized things that have two sides - a good one and a bad one.

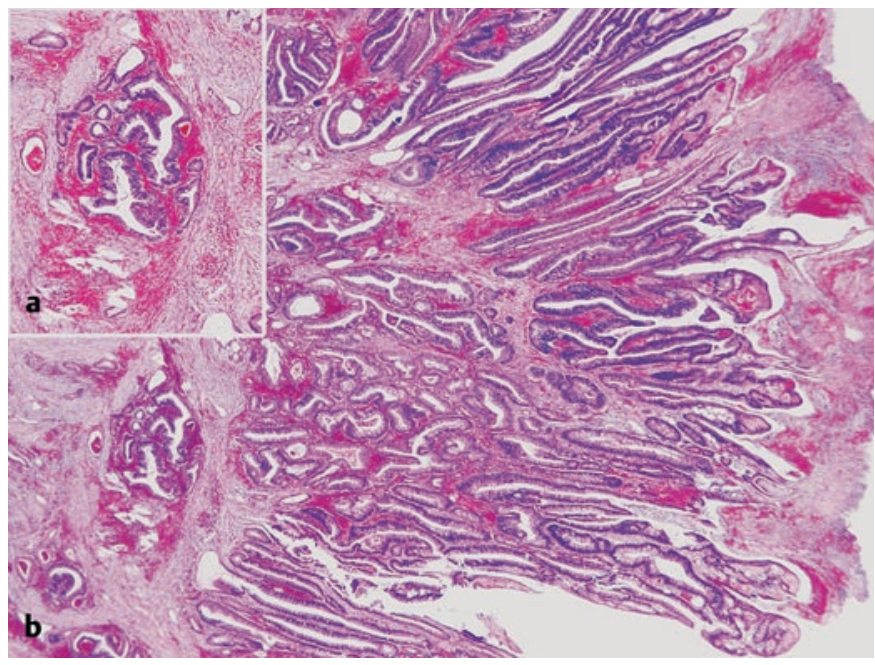

Figure 2 Microscopic examination of the first head showed that it was a highly differentiated adenocarcinoma with neoplastic glands in the muscularis mucosae (a), arising within an adenoma (b). There was a focus of invasion surrounded by desmoplasia (a). Periodic acid-Schiff, original magnification $\times 200(\mathbf{a}), \times 16(\mathbf{b})$.

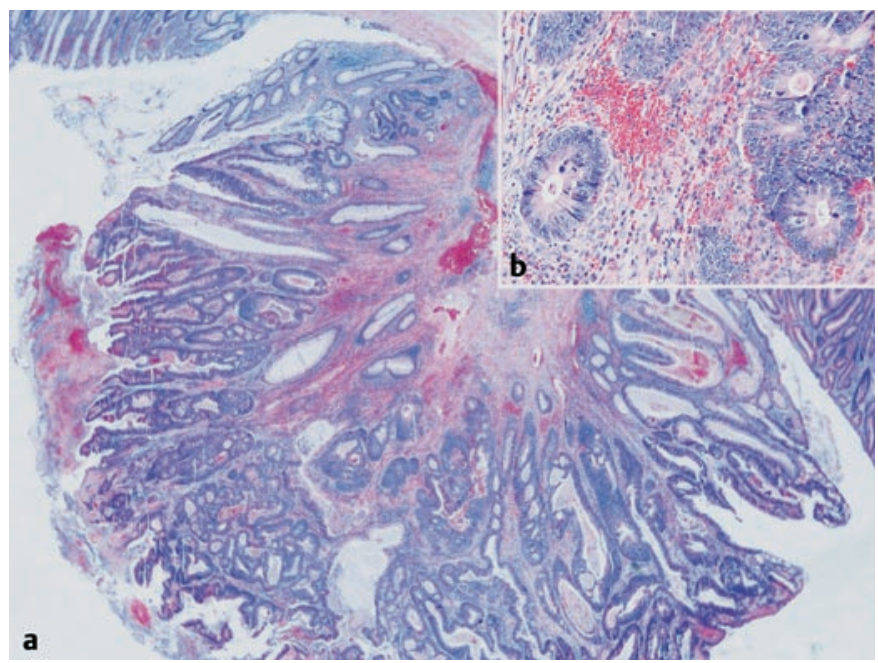

Figure 3 The second head consisted of a tubulovillous adenoma with high-grade dysplasia. The adenoma consisted of dysplastic tubular crypts, as well as finger-like structures, and had a glandwithin-gland appearance without intervening stroma. $\mathbf{b}$ The dysplastic epithelium, with enlarged and stratified nuclei, extending into the upper third of the cells, with loss of polarity. Hematoxylin-eosin, original magnification $\times 200$ (a), $\times 16$ (b).

\section{S. Gölder ${ }^{1}$, F. Bataille ${ }^{2}$, J. Schölmerich ${ }^{1}, \quad$ Corresponding Author \\ G. Rogler ${ }^{1}$}

${ }^{1}$ Dept. of Internal Medicine I, University of Regensburg, Regensburg, Germany

${ }^{2}$ Dept. of Pathology, University of Regensburg, Regensburg, Germany

\section{G. Rogler, M.D., Ph. D.}

Dept. of Internal Medicine I University Regensburg 93042 Regensburg Germany

Fax: $\quad$ +49-941-944 7073

E-mail: gerhard.rogler@klinik.uniregensburg.de 